\title{
Platelet-rich plasma improves chronic inflammatory pain by inhibiting PKM2-mediated aerobic glycolysis in astrocytes
}

\author{
Xiang Wei", Xiao-Hong Jin", Xiao-Wen Meng", Jie Hua, Fu-Hai Ji, Li-Na Wang, Jian-Ping Yang \\ Department of Anesthesiology and Pain Management, the First Affiliated Hospital of Soochow University, Suzhou, China \\ Contributions: (I) Conception and design: LN Wang; (II) Administrative support: None; (III) Provision of study materials or patients: None; (IV) \\ Collection and assembly of data: X Wei, XH Jin, XW Meng; (V) Data analysis and interpretation: X Wei, XH Jin, XW Meng, J Hua, FH Ji, JP Yang; \\ (VI) Manuscript writing: All authors; (VII) Final approval of manuscript: All authors. \\ \#These authors contributed equally to this work. \\ Correspondence to: Jian-Ping Yang; Li-Na Wang. Department of Anesthesiology and Pain Management, the First Affiliated Hospital of Soochow \\ University, Suzhou, China. Email: szyangjp@126.com; Wangln@suda.edu.cn.
}

Background: Astrocytes are highly glycolytic cells that play a crucial role in chronic pain. Recently it has been found that inflammation and metabolism are related to the inflammatory stimuli closely that cause cellular metabolic changes. Pyruvate kinase M2 (PKM2) is a critical metabolic kinase in aerobic glycolysis or the Warburg effect. Besides, it also plays a crucial role in cell proliferation and signal transduction, but its role in astrocytes is still unclear.

Methods: The chronic inflammatory pain model was set up by intraplantar injection of complete Freund's adjuvant (CFA) in Sprague Dawley (SD) rats as well as the cell model was constructed by lipopolysaccharidetreated primary astrocytes. Von Frey filament stimulation was used to continuously observe the changes of pain behavior in rats after modeling. Then, immunofluorescence staining and Western blot tests were used to observe the expression levels of glial fibrillary acidic protein (GFAP), pyruvate kinase (PKM2), signal transducers and activators of transcription 3 (STAT3) and high mobility group box-1 protein (HMGB1). After that, specific kits measured lactate contents. Finally, we observed the platelet-rich plasma's (PRP) effect on mechanical hyperalgesia in rats with inflammatory pain induced by CFA and its effect on related signal molecules.

Results: We found that in the CFA-induced inflammatory pain model, astrocytes were significantly activated, GFAP was increased, PKM2 was significantly up-regulated, and the glycolytic product lactate was increased. Also, intrathecal injection of PRP increased the pain threshold, inhibited the activation of astrocytes, and decreased the expression of PKM2 and aerobic glycolysis; in LPS-activated primary astrocytes as an in vitro model, we found PKM2 translocation activationSTAT3 signaling resulted in sustained activation of astrocyte marker GFAP, and the expression level and localization of p-STAT3 were correlated with PKM2. PRP could inhibit the activation of astrocytes, reduce the expression of PKM2 and the expression levels of glycolysis and GFAP, GLUT1, and p-STAT3 in astrocytes.

Conclusions: Our findings suggest PKM2 not only plays a glycolytic role in astrocytes, but also plays a crucial role in astrocyte-activated signaling pathways, and PRP attenuates CFA induced inflammatory pain by inhibiting aerobic glycolysis in astrocytes, providing a new therapeutic target for the treatment of inflammatory pain.

Keywords: Platelet-rich plasma (PRP); chronic inflammatory pain; aerobic glycolysis; pyruvate kinase M2 (PKM2)

Submitted Sep 07, 2020. Accepted for publication Oct 19, 2020.

doi: 10.21037/atm-20-6502

View this article at: http://dx.doi.org/10.21037/atm-20-6502 


\section{Introduction}

Chronic pain is a common disease in clinical practice, but there is no effective treatment, of which inflammatory pain is the most representative type. Inflammatory pain is a pathological pain induced by tissue inflammatory response due to infection, trauma, chemical stimulation, and other causes, with spontaneous pain, hyperalgesia, and abnormal pain at the injury site, which can also cause secondary pain in peripheral tissues (1). Persistence of inflammatory pain can cause pain and emotional abnormalities, and even lead to other serious complications (2). Thus, it is vital to give certain drugs to relieve pain for improving the quality of life and stabilize patients' moods.

The spinal dorsal horn is the site of the primary integration site of nociceptive information transmission, and neuron-glial cell interactions are critical to developing and progressing chronic pain (3). Recent studies have shown that glial cells play an essential role in the occurrence and development of neuropathic pain $(4,5)$. Activation of glial cells and the production of inflammatory mediators are primary events in neuroinflammation (6). Synaptic remodeling and central sensitization mediated by glial cells and inflammatory mediators are essential for neuropathic pain (7). Recent studies have found that metabolic reprogramming plays a crucial role in immune cell responses (8). Under sufficient oxygen, cells still consume a considerable amount of glucose and support active glycolysis. This abnormal metabolic mode is called aerobic glycolysis (9). Studies have shown that activation of immune cells in response to inflammatory stimuli also shows a shift in aerobic glycolysis as the primary functional mode. Compared with the oxidative phosphorylation of mitochondria, although the glycolysis pathway is inefficient in the utilization of glucose, it can generate more energy per unit time in the process of immune cell biosynthesis to adapt to the rapidly increasing ATP demand (10). Like immune cells, when stimulated by inflammation, activated astrocytes switch from predominantly oxidative phosphorylation to predominantly aerobic glycolysis to meet biosynthetic needs and produce sufficient energy (11). Astrocytes are highly glycolytic cells and the primary site of glycolytic activity in the central nervous system, and they supply energy substrates to neurons through a lactic acid shuttle. Enhanced glycolysis in cells provides a biosynthetic barrier for pro-inflammatory factors (12). Glial cells may synthesize and secrete many inflammatory factors and cytokines through aerobic glycolysis, which can combine with the corresponding receptors of neurons and enhance the excitability of neurons, thus playing a critical role in chronic pain

Pyruvate kinase, a rate-limiting enzyme of the glycolytic pathway, can catalyze the formation of pyruvate and ATP from phosphoenolpyruvate and $\operatorname{ADP}(13,14)$, and pyruvate kinase M2 (PKM2) is highly expressed in astrocytes (15). The metabolism of cells must match their functional needs. In chronic pain, activated glial cells will release inflammatory factors and cytokines to bind to neurons' corresponding receptors, so they need more energy (14). The conversion from oxidative phosphorylation to glycolytic metabolism can better adapt to this metabolic need (16). It is well-known that PKM2 plays a vital role in the final stage of glycolysis, regulating the synthesis of lactate, affecting the intracellular environment $\mathrm{pH}$ and cellular function (17). Under pathological conditions, lactate accumulation leads to a decrease in intracellular PH and tissue acidification, resulting in pain; therefore, PKM2 is considered playing a pivotal role in pain initiation or modulation. With the deepening of research, the researchers also found that increased glucose uptake and glycolytic flux can promote the production of mitochondrial reactive oxygen species, which in turn promote the dimerization of PKM2 and translocate it into the nucleus, combining with various transcription factors, and aggravating the inflammatory reaction. For example, p300 acetyltransferase can catalyze PKM2 acetylation into the nucleus and bind to histone 3 (H3) and signal transducer and activator of transcription (STAT3) (18); EGF can induce PKM2 to enter the nucleus, phosphorylate tyrosine at 333 aa of $\beta$-Catenin, and promote the binding of PKM 2 and $\beta$-catenin, thereby enhancing cell proliferation and subsequent transcription of related genes (19). Nuclear PKM2 enhances STAT3 transcriptional activity through phosphorylating STAT3 and leads to the transactivation of MEK5; up-regulation of MEK5 can further promote PKM2-mediated cell proliferation (20). These signaling pathways activate spinal glial cells after nerve injury and promote neuropathic pain (21). Taga et al. (22) suggested that the activation of astrocytes depends largely on IL-6 family cytokine-mediated STAT3 activation due to STAT3 binding elements in the promoter region of the astrocyte marker GFAP gene (23). Although PKM2 plays a crucial role in metabolism, gene transcription, and inflammation, whether it is involved in chronic inflammatory pain by regulating astrocytes remains unknown.

It has recently been reported that platelet-rich plasma 
(PRP) can relieve pain in patients with a tendon injury, but the mechanism of this effect is unknown. PRP is a supernatant containing various growth factors that are involved in the healing of damaged tissues by simply centrifuging the whole blood and removing red blood cells, which are involved in the healing of damaged tissues (24), and can regulate cellular processes including chemotaxis, angiogenesis, mitosis, differentiation, and metabolism (25). As an emerging adjuvant therapy in surgical treatment, PRP has been widely used in clinical practice (26). Whether PRP has an analgesic effect on inflammation-induced pain has not been reported.

In this study, we analyzed the expression of GFAP, PKM2, STAT3, and HMGB1 after intraplantar injection of complete Freund's adjuvant (CFA) in rats. In this study, we investigated the role of PKM2 in LPS-stimulated aerobic glycolysis in primary astrocytes, we hypothesized that PRP effectively inhibited the inflammatory response and HMGB1 expression by regulating PKM2-mediated glycolysis and STAT3 signaling-mediated astrocyte activation, thereby alleviating CFA induced pain in rats, which is essential for clinical treatment, to find new targets for inflammatory pain treatment.

We present the following article in accordance with the ARRIVE reporting checklist (available at http://dx.doi. org/10.21037/atm-20-6502).

\section{Methods}

\section{Animal model and intrathecal administration}

The Animal Experimental Ethics Committee approved the experimental protocol and surgical procedures of Soochow University (No.: 2017-185). All experiments were performed following the National Institutes of Health Guide for the Care and Use of Laboratory Animals and the guidelines of the International Association for the Study of Pain. CFA was injected into the plantar subcutaneously to construct a rat model of inflammatory pain. Healthy, male Sprague-Dawley (SD) rats weighing $200 \pm 20 \mathrm{~g}$ and neonatal rats (aged 1-2 days)were used in this study and acquired from Shanghai Slack Laboratory Animal Company. They were kept under a controlled condition (temperature of $24-26{ }^{\circ} \mathrm{C}$, relative humidity $40-60 \%, 12 \mathrm{~h}$ light-dark cycle; free access to food and water). After isoflurane anesthesia, $100 \mu \mathrm{L}$ CFA was injected into the sole of left hind foot in the CFA group, and the same volume of normal saline was injected into the control (CON) group.
Intrathecal administration was performed as previously described (27). Briefly, SD rats were anesthetized and placed in a prone sitting position. The L4-5 spinous process space was used as the puncture point. After probing the hip tubercle with the left hand, the thumb and middle finger were placed on both sides of the L4-5 spinous process space of rats and were positioned with the index finger; The microsyringe was held in the right hand to insert the needle vertically from the space slowly. When the tail-flick action occurred, the syringe was withdrawn with cerebrospinal fluid and then injected. After injection, the tail-flick action often occurred, which was a successful intrathecal administration.

\section{Experimental design}

This experiment includes two parts: in vitro and in vivo experiments. A computer-generated random table was used to divide cells or rats into different groups. All experiments in this study were independently repeated 3 times.

\section{In vivo experiment}

- Part 1: rats were randomly divided into CON and CFA groups. $100 \mu \mathrm{L}$ CFA was injected into the metatarsals of the left hind foot of CFA, and the CON group was injected with the same amount of saline.

- Part 2: to investigate the role of astrocytes in CFA induced inflammatory pain, the role of molecules involved in astrocyte activation in CFA induced inflammatory pain was assessed. SD rats were randomly divided into two groups: $\mathrm{CON}$ and CFA group.

- Part 3: to investigate the role of PRP in inflammatory pain. Rats were randomly divided into three groups: CON, CFA and CFA + PRP group (intrathecal injection of $20 \mu \mathrm{L}$ PRP).

* Part 4: to investigate the role of PKM2 in inflammatory pain, PKM2-IN-1, a specific inhibitor of PKM2, was assessed. Rats were randomly divided into three groups: CON, CFA and CFA + IN-1 group (intrathecal injection of $20 \mu \mathrm{L}$ PKM2-IN-1).

\section{In vitro experiment}

* Part 5: neonatal rat primary astrocytes were randomly divided into CON and three LPS groups, and CON group received normal culture. At LPS, they were treated with $(10,100,1,000 \mathrm{ng} / \mathrm{mL})$ LPS for $12 \mathrm{~h}$. Next to investigate the effect of PRP on LPS-induced primary astrocytes injury. Cells were randomly divided into three groups: CON, LPS and LPS + PRP group 
(incubated with $10 \%$ PRP for $2 \mathrm{~h}$ before $100 \mathrm{ng} / \mathrm{mL}$ LPS treatment).

- Part 6: to investigate whether PKM2 can continuously activate astrocytes by activating STAT3. The cells were randomly divided into three groups: CON, LPS and LPS + IN-1 group (incubated with 3 $\mu \mathrm{mol} / \mathrm{L}$ PKM2-IN-1 for $2 \mathrm{~h}$ before $100 \mathrm{ng} / \mathrm{ml} \mathrm{LPS}$ treatment).

\section{Pain behavior}

Mechanical thresholds of plantar pain sensation in rats were measured with the von Frey filaments (28). In other words, rats were individually placed in a clear Plexiglas box before the experiment, allowed to acclimate to the environment for 15 minutes, and then a series of the von-Frey filaments with increasing pressure $(2.0,4.0,6.0,8.0,10.0,15.0$, and $26.0 \mathrm{~g}$ ) were applied to the plantar surface of the right hind paw until the rats exited the paw. $1.0 \mathrm{~g}$ of filaments were applied vertically from below the mesh to stimulate the plantar surface, and each trial lasted 5 to 6 seconds. We recorded the force applied upon withdrawal, and the response was expressed in grams. Set the cut-off threshold at 26 g. Stimulation was performed in an up-down method, and results were converted to values that represent a $50 \%$ withdrawal threshold.

\section{Hematoxylin and eosin (H\&E) staining}

Rats were deeply anesthetized with pentobarbital and were transcardially perfused with $37^{\circ} \mathrm{C}$ normal saline followed by $4 \%$ paraformaldehyde in $0.1 \mathrm{M} \mathrm{PBS}\left(\mathrm{pH}, 7.4 ; 4^{\circ} \mathrm{C}\right)$. Then, the tissues were removed at once and fixed in $10 \%$ buffered formalin for 48 hours and prepared for routine paraffin histology. The paraffin-embedded 5 - $\mu$ m-thick sections from separate groups were stained by $\mathrm{H} \& \mathrm{E}$.

\section{Primary astrocyte culture}

Newborn (1-2 days) Sprague-Dawley rats were disinfected with $75 \%$ ethanol solution, decapitated, and the collected cortex was cut into fragments, digested with $0.25 \%$ trypsin for 20 minutes, centrifuged at 1,500 rpm for 10 minutes, and the supernatant was discarded. The cells were washed three times with PBS solution, suspended in DMEM/ F12 medium, and seeded on a culture dish to grow until fusion. Astrocytes were purified by shaking at $37^{\circ} \mathrm{C}$ for 8 hours. Purified astrocytes were cultured in the DMEM/
F12 culture medium at $37^{\circ} \mathrm{C}$ in an incubator with $5 \% \mathrm{CO}_{2}$.

\section{Preparation of PRP}

Under aseptic conditions, a $10 \mathrm{~mL}$ sterile syringe was flushed with $1 \mathrm{~mL}$ of $10 \%$ sodium citrate and then used to withdraw $10 \mathrm{~mL}$ of blood for venipuncture under direct vision in anesthetized rats. The blood was placed into a $15 \mathrm{~mL}$ centrifuge tube. Then, the PRP was extracted following the Aghaloo method (29), and platelets were counted and stored in a refrigerator at $-80^{\circ} \mathrm{C}$ for future use. It is activated with a mixture of thrombin and $10 \%$ calcium chloride before use.

\section{Immunofluorescence}

For double immunofluorescence, the frozen sections were rinsed with $0.01 \mathrm{~mol} / \mathrm{L}$ PBS for $3 \times 10$ minutes and then sealed with a blocking buffer containing $5 \%$ BSA and $0.1 \%$ Triton X-100 at room temperature (RT) for 2 hours. Then, monoclonal antibody (Ab) mouse anti-GFAP (1:400, \#3670, Cell Signaling Technology), rabbit anti-PKM2 (1:400, 15822-1-AP, Proteintech) were added. PBS was used instead of the primary $\mathrm{Ab}$ as a negative control and incubated overnight at $4{ }^{\circ} \mathrm{C}$. After rinsing with $0.01 \mathrm{~mol} / \mathrm{L}$ PBS for $3 \times 10$ minutes, Alexa Fluor ${ }^{\circledR}$ 488-labeled donkey anti-mouse secondary Ab and Alexa Fluor ${ }^{\circledR}$ 594-labeled goat anti-rabbit secondary Ab was added, respectively. The slides were incubated at RT for 2 hours and then rinsed with $0.01 \mathrm{~mol} / \mathrm{L}$ PBS for $3 \times 10$ minutes. The slides were mounted in the dark, dried with a fluorescent sealing agent, and photographed under a fluorescent confocal microscope.

\section{Western blot}

The procedure was like the method described previously (30). The rats were killed under anesthesia. About $100 \mathrm{mg}$ of lumbar spinal cord tissue was cut into pieces. After adding protein lysate into a homogenizer, the homogenate was ground, and centrifuged supernatant was the total protein extracted. Then we used the BCA method to determine the protein concentration of each group. Each sample was loaded with $30 \mu \mathrm{g}$ and boiled with a loading buffer for 15 minutes. Then $10 \%$ of SDS-PAGE gel electrophoresis was used to isolate the protein and transferred to the PVDF membrane. The $5 \%$ skim milk powder was sealed at RT for two hours. Then the first Ab (anti GFAP 1:1,000, \#3670, Cell Signaling Technology, anti-p-STAT3 1:1,000, \#9145, 
A

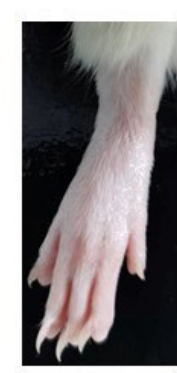

C

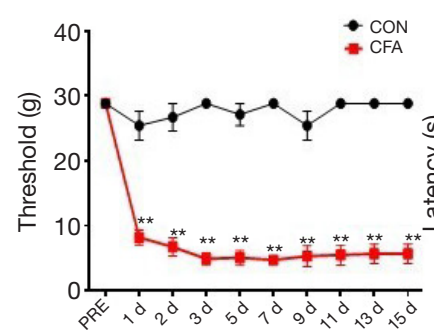

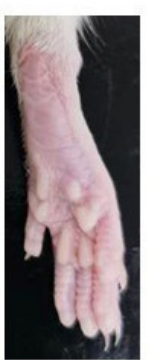

B

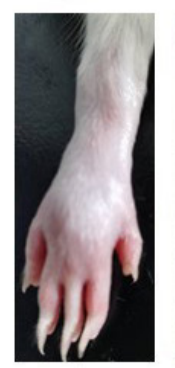

D

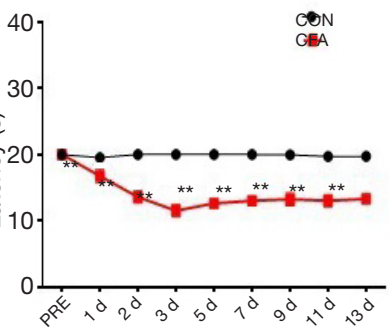

$\mathrm{E}$

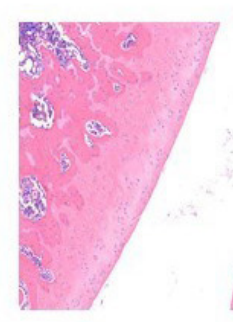

G
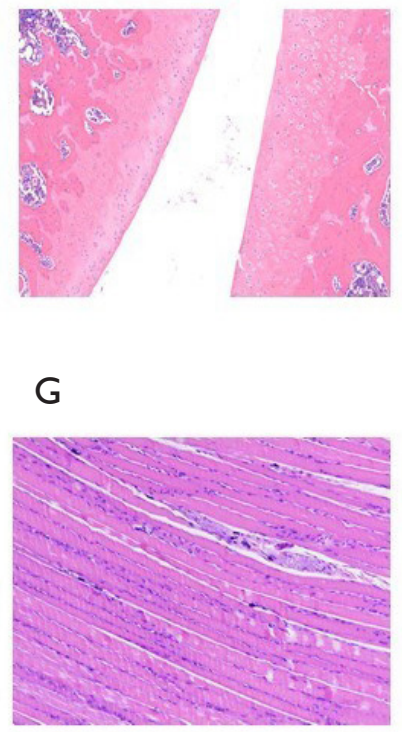

$\mathrm{F}$
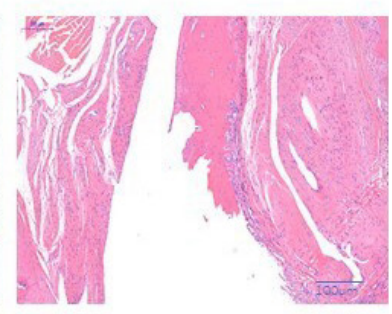

$\mathrm{H}$

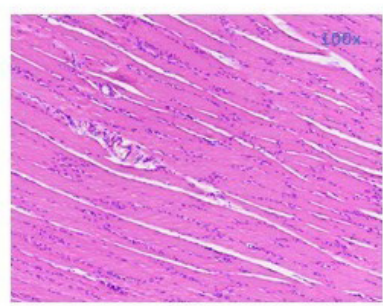

Figure 1 Morphological, behavioral, and histochemical analyses of CFA model rats. (A,B) Hindfoot morphological changes (anterior and posterior views) in CON and CFA rats. (C,D) Mechanical and thermal hyperalgesia in the hindfoot of CON and CFA rats. (E,F) Histopathological images of hematoxylin-eosin (H\&E) staining of knee joint specimens from rats in the two groups (G,H), hematoxylineosin (H\&E) stained histopathological images of plantar muscle specimens from rats in both groups. The data are presented as the mean \pm SEM in all figures and throughout the text. CFA, complete Freund's adjuvant.

Cell Signaling Technology, anti-PKM2 1:1,000, 158221-AP, Proteintech, anti-GLUT1, 1:1,000, 21829-1-AP, Proteintech, anti-Lamin B1 1:1,000, MULTISCIENCES, anti- $\beta$-actin 1:1,000, MULTISCIENCES) were incubated overnight at $4{ }^{\circ} \mathrm{C}$. On the second day, TBST was used to wash the membrane $3 \times 10$ minutes, and the corresponding horseradish peroxidase-labeled secondary $\mathrm{Ab}(1: 4,000)$ was added respectively, incubated on the shaker for 2 hours. Finally, ECL chromogenic fluid was added for exposure, and the image was taken and analyzed in the gel imaging system, and each band was calculated with Image J software.

\section{Lactic acid test}

Rat L4-5 spinal cords, as well as treated cell culture media, were collected, samples were evaluated according to the manufacturer's instructions, and lactate levels were normalized to control samples. (Lactic Acid assay kit, A0192-1, Nanjing Jiancheng).

\section{Statistical analysis}

Results are expressed as mean \pm SEM. The withdrawal thresholds between separate groups over time were evaluated with two-way analysis of variance (ANOVA) followed by Bonferroni post hoc tests. For Western blot and immunohistochemistry assays, data were analyzed using the two-tailed $t$-test for the two groups' comparison and oneway ANOVA for multi-group comparisons.

\section{Results}

\section{Establishment of an animal model of CFA inflammatory pain (Figure 1)}

Mononuclear arthritis developed within 72 hours after SD rats received $100 \mu \mathrm{L}$ CFA. After injection of CFA, swelling and enlargement appeared on the third day after injection (Figure 1B). However, the CON group injected with saline showed no significant size change (Figure 1A). Mechanical hyperalgesia and thermal hyperalgesia were assessed in the animals' hind limbs to assess the chronic pain in the CFA model (Figure $1 C, D$ ). In the $\mathrm{CON}$ group, there was no difference in the pain threshold of the hind paw at all time points, but in the CFA model, CFA rats were lower than the $\mathrm{CON}$ group at most time points and reached the lowest 
level on day3, suggesting that CFA caused hindfoot pain in rats $\left(\mathrm{n}=8 .{ }^{*}, \mathrm{P}<0.05,{ }^{* *}, \mathrm{P}<0.01\right.$, compared with the $\left.\mathrm{CON}\right)$. The pathological results of the knee joint (Figure 1E,F) showed that the chondrocytes in the knee joint of rats in the CON group were normal. The chondrocytes were closely arranged, the thickness of the cartilage layers was standard, there was no cartilage loss, and the bone structure and morphology were tight and regular. Further, the knee joint surfaces of rats in the CFA group were damaged, showed shedding, articular cartilage necrosis, extensive meniscus loss, subchondral bone sclerosis, bone sparsely fragmented, and the structure was disordered. Pathological results of muscle tissue (Figure $1 G, H$ ). In the CON group, the structure of muscle fibers was clear, and the muscle fibers were arranged neatly with uniform intervals; in the CFA group, many inflammatory cells infiltrations were observed, muscle fibers were disorganized, and the thickness of the intervals varied. The above results show that the CFA model was successfully set up.

\section{Activation of astrocytes in spinal dorsal horn induced by CFA (Figure 2)}

Unilateral injection of $100 \mu \mathrm{L}$ CFA into a hindpaw of rats produces rapid and persistent

inflammatory pain. To detect the activation of astrocytes, we collected the spinal cord (L4-6) 7 days after CFA or saline injection. As shown in Figure 2, we detect the expression of GFAP, PKM2, p-STAT3, and HMGB1 was studied in the spinal cord of the CON group and CFA group. Immunofluorescence staining showed that compared with the CON group, GFAP, PKM2, p-STAT3, and HMGB1 in the CFA group increased significantly on the 7 th day after plantar injection of CFA, and the activated astrocytes became larger and thicker. The immunofluorescence intensity of GFAP, PKM2, p-STAT3, and HMGB1 increased (Figure 2A,C,E,G). Besides, Western blot analysis further showed CFA significantly increased GFAP, PKM2, p-STAT3, and HMGB1 protein levels in the spinal dorsal horn on day 7 (Figure 2B,D,F,H). These results suggest CFA induces the activation of astrocytes in the dorsal horn.

\section{Intrathecal injection of PRP can reduce mechanical analgesia caused by CFA (Figure 3)}

If astrocyte activation can lead to pain, then the intervention of astrocytes can reduce pain hypersensitivity. $20 \mu \mathrm{L}$ PRP was injected intrathecally (31) daily for seven days starting from 3 days after surgery to test whether PRP ameliorates CFA induced pain by inhibiting astrocyte activation. Intrathecal administration of PRP can increase the mechanical pain threshold in CFA rats compared with the CON group (Figure 3A); Meanwhile, to determine whether the analgesic effect of PRP acts through inhibition of spinal astrocytes. In immunofluorescence staining analysis, the results indicated the expression of GFAP and PKM2 was significantly enhanced in the spinal dorsal horn of CFA rats on day 7, and the level of PKM2 in the spinal dorsal horn was significantly reduced in the CFA+PRP group compared with CFA group after PRP treatment, especially in astrocytes (Figure 3B). Western blot analysis further showed that CFA+PRP resulted in a significant reduction in PKM2 protein levels in the spinal dorsal horn on day 7. Immunofluorescence data were further confirmed in Western blot analysis. Since the STAT3 pathway is involved in spinal astrocyte activation and neuroinflammation under several pathological pain conditions, the expression of p-STAT3 andHMGB1 in the spinal dorsal horn was tested in different groups on day seven after modeling. Western blot analysis showed CFA resulted in a significant upregulation of p-STAT3. This upregulation of STAT3 signaling was reversed after PRP injection (Figure $3 C,{ }^{*} \mathrm{P}<0.05$ compared with the CON group).

\section{Intrathecal injection of PKM2-IN-1 can alleviate the mechanical byperalgesia induced by CFA (Figure 4)}

To investigate whether astrocyte activation by CFA is related to the glycolytic pathway, we detected a significant increase in the expression of PKM2 in the spinal dorsal horn of the CFA model group, so whether inhibition of PKM2 reduces astrocyte activation thereby improving CFA induced pain, We intrathecally injected $20 \mu \mathrm{L}$ PKM2$\mathrm{IN}-1$, an inhibitor of PKM2, from 5 to 7 days after surgery. The results indicated that intrathecal administration of PKM2-IN-1 could increase the mechanical pain threshold in CFA rats compared with the CON group (Figure $4 A$ ); CFA induced a significant increase in lactate level in L4-6 spinal cord $(\mathrm{P}<0.05)$. PRP and PKM2-IN-1 inhibited the increased lactate level $(\mathrm{P}<0.05)$ (Figure 4B). Glucose transporter 1 (GLUT1) expression was quantified using immunoblotting to investigate potential causes of the increased lactate. Western blot analysis further showed CFA + PKM2-IN-1 resulted in a significant decrease in GFAP and PKM2 protein levels in the spinal dorsal horn on day 
A

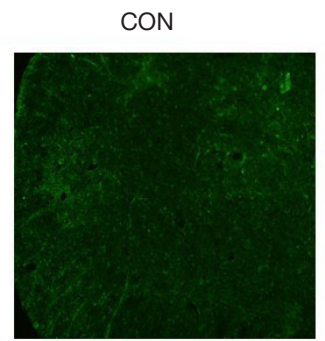

B
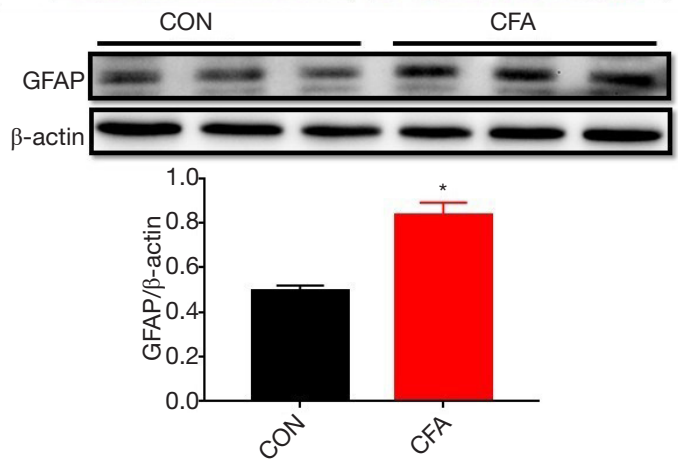

E

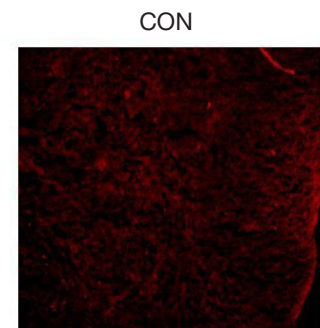

F
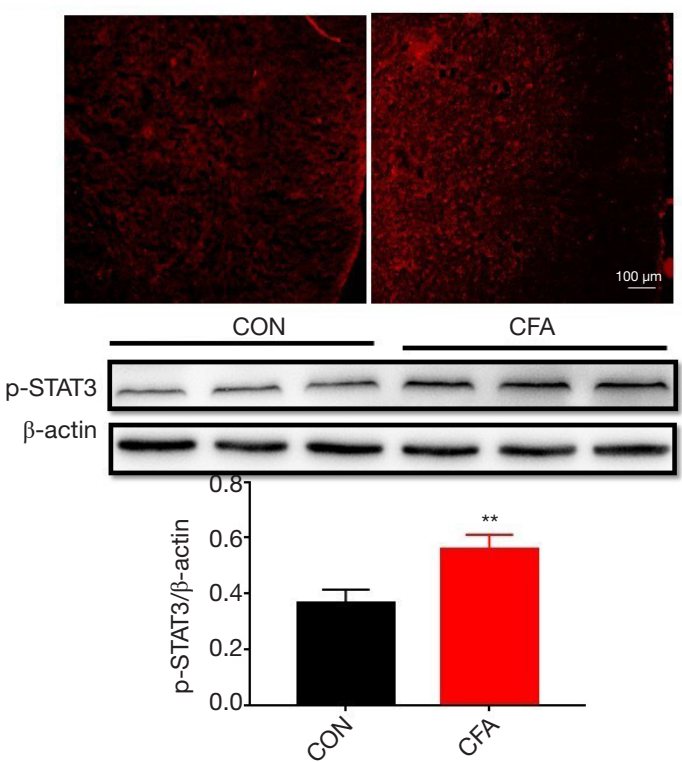

C

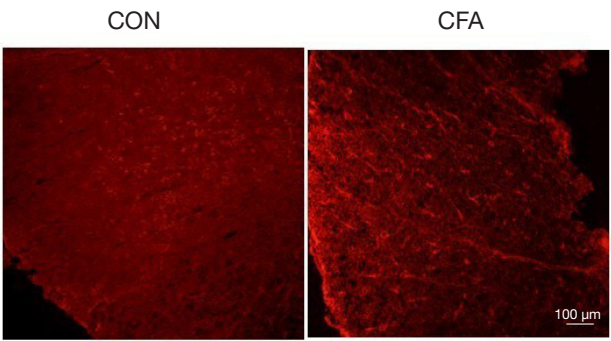

D

CON
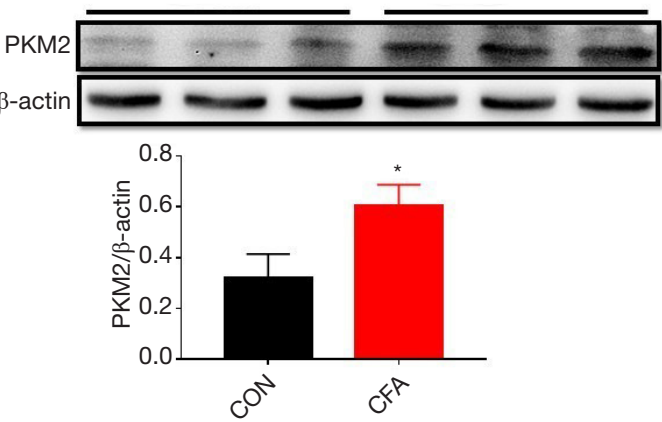

G

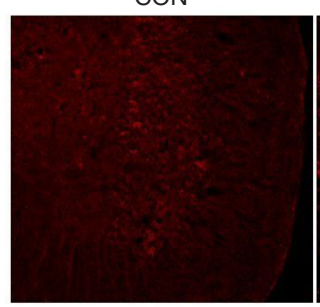

CON

$\mathrm{H}$

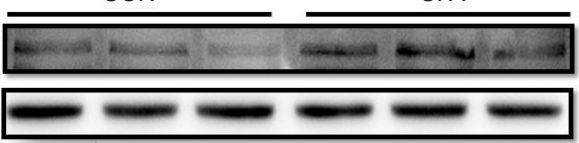

$\beta$-actin

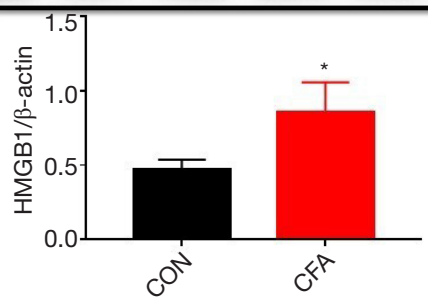

Figure 2 CFA induces the expression of GFAP, PKM2, p-STAT3, and HMGB1 protein in the spinal cord. (A) Immunostaining shows the expression of GFAP in the spinal cord of CON and CFA rats. GFAP was enhanced at seven days after CFA. $n=3$ rats/group. (B) Western blot shows the increase of GFAP protein expression in the spinal cord after CFA injection. $\mathrm{n}=3$ rats/group. (C) Immunostaining shows the expression of PKM2 in the spinal cord of CON and CFA rats. PKM2 was enhanced at seven days after CFA. $\mathrm{n}=3$ rats/group. (D) Western blot shows the increase of PKM2 protein expression in the spinal cord after CFA injection. $n=3$ rats/group. (E) Immunostaining shows the expression of p-STAT3 in the spinal cord of CON and CFA rats. p-STAT3 was enhanced at seven days after CFA. $\mathrm{n}=3$ rats/group. (F) Western blot shows the increase of p-STAT3 protein expression in the spinal cord after CFA injection. $\mathrm{n}=3$ rats/group. (G) Immunostaining shows the expression of HMGB1 in the spinal cord of CON and CFA rats. HMGB1 was enhanced at seven days after CFA. Scale bar: $100 \mu \mathrm{m}$. $\mathrm{n}=3$ rats/group. (H) Western blot shows the increase of HMGB1 protein expression in the spinal cord after CFA injection. $\mathrm{n}=3$ rats/group. *, $\mathrm{P}<0.05$ compared to $\mathrm{CON}$; **, $\mathrm{P}<0.01$ compared to CON. CFA, complete Freund's adjuvant. 
A

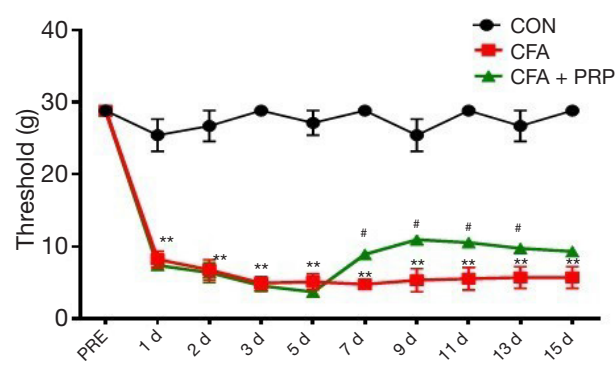

B

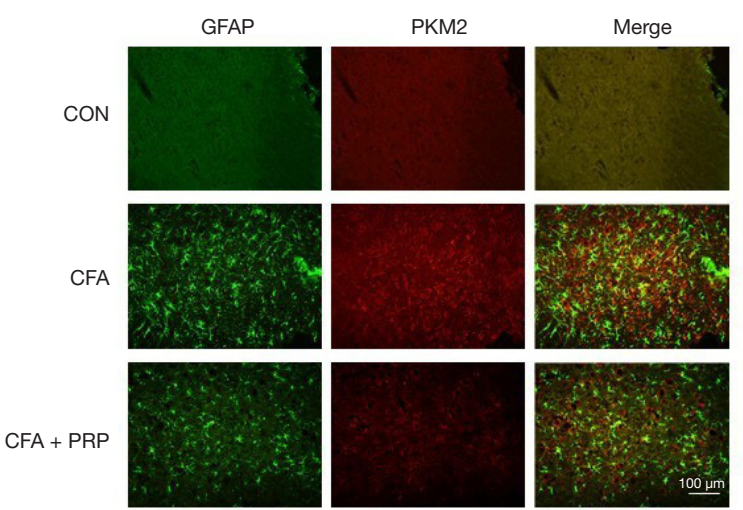

C
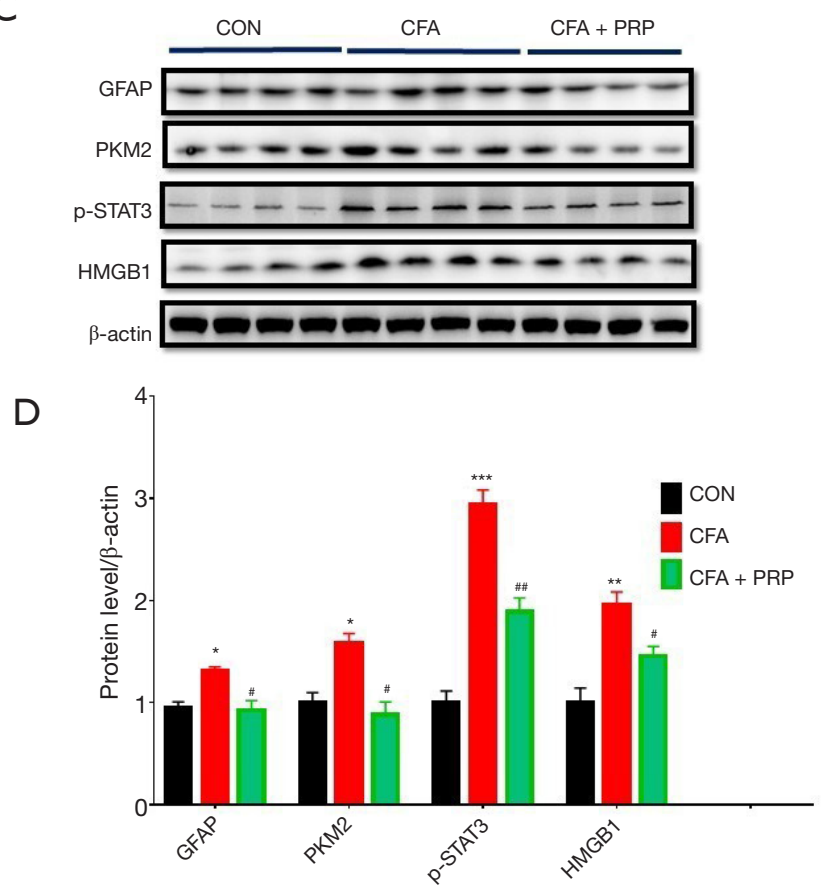

Figure 3 Intrathecal injection of PRP reduced CFA induced mechanical hyperalgesia and the expression of related proteins. (A) Intrathecal injection of $20 \mu \mathrm{L}$ PRP reversed mechanical hyperalgesia induced by $\mathrm{CFA}$, **, $\mathrm{P}<0.01$ compared with $\mathrm{CON}$, ${ }^{*}, \mathrm{P}<0.05$ compared with $\mathrm{CFA}$, $\mathrm{n}=6$ rats/group. (B) Immunofluorescence shows that PRP inhibits the expression of GFAP (green) and PKM2 (red). Scale bar: $100 \mu \mathrm{m}$. N=3. (C.D) Western blot showed that PRP partially reversed the CFA induced increase in GFAP, PKM2, p-STAT3, HMGB1 protein levels in the spinal cord. $\mathrm{N}=4$. *, $\mathrm{P}<0.05$, **, $\mathrm{P}<0.01$, ***, $\mathrm{P}<0.001$, compared with $\mathrm{CON},{ }^{*}, \mathrm{P}<0.05,{ }^{\#}, \mathrm{P}<0.01$ compared with $\mathrm{CFA}$. $\mathrm{PRP}$, platelet-rich plasma; CFA, complete Freund's adjuvant.

7 , as well as a reversal of the upregulation of p-STAT3, GLUT1, and HMGB1 (Figure $4 C, D,{ }^{*} \mathrm{P}<0.05$ compared with the $\mathrm{CON}$ ).

\section{PRP inbibits LPS-induced glycolytic in primary astrocytes (Figure 5)}

To further investigate the relationship between PKM2mediated glycolysis and astrocyte activation, we used an astrocyte inflammatory model cultured in vitro. The purified cells after repeated passages were identified by GFAP and DAPI immunofluorescence double labeling. The astrocytes were then stained with DAPI, and the nucleus was highly specific, presenting with blue fluorescence and a discernible nucleus (Figure $5 A$, a). The GFAP-labeled positive cells had large and irregular cell bodies and were interlaced to form a reticular structure, presenting significant green fluorescence (Figure $5 A, \mathrm{~b}$ ). The purity of astrocytes was more than $95 \%$, indicating that the cultured cells were SD rat astrocytes (Figure $5 A$, c) and could be used for subsequent experiments. Scale bars: 40 (top) 100 (middle), and $200 \mu \mathrm{m}$ (bottom) Astrocytes were then stimulated with different concentrations of LPS (10, $100,1,000 \mathrm{ng} / \mathrm{mL}$ ) to mimic the stimulatory effect of inflammation on astrocytes. GFAP, PKM2, p-STAT3, and GLUT1 levels were significantly increased by $100 \mathrm{ng} / \mathrm{mL}$ LPS treatment, indicating that PKM2-mediated glycolysis is related to post-LPS stimulation (Figure $5 B, C$ ). However, the release of lactic acid did not increase (Figure $5 D$ ). These results show the glycolytic of astrocytes increase after LPS stimulation. Next, we studied whether PRP exerts its effect on astrocytes; we employed PRP to treat LPSstimulated astrocytes in vitro. The expression changes of PKM2 protein in astrocytes after LPS and PRP treatment were detected by fluorescence. Immunofluorescence results showed PKM2 was present in the CON astrocytes. After stimulation with LPS, the expression of PKM2 protein was significantly increased, while after treatment with 
A

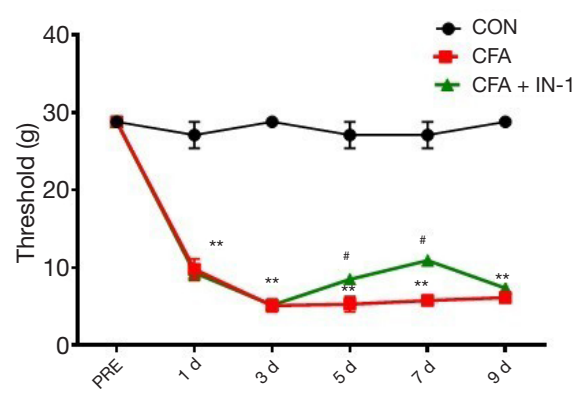

C

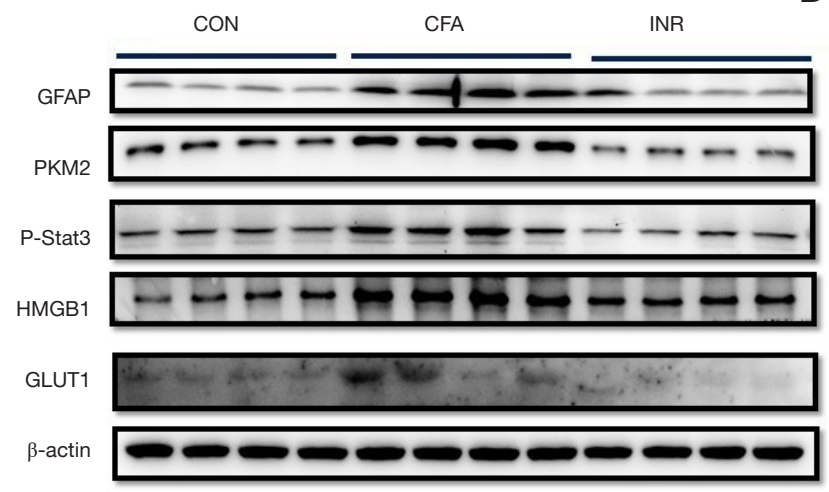

B

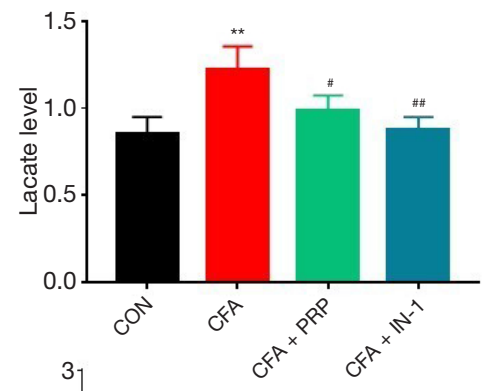

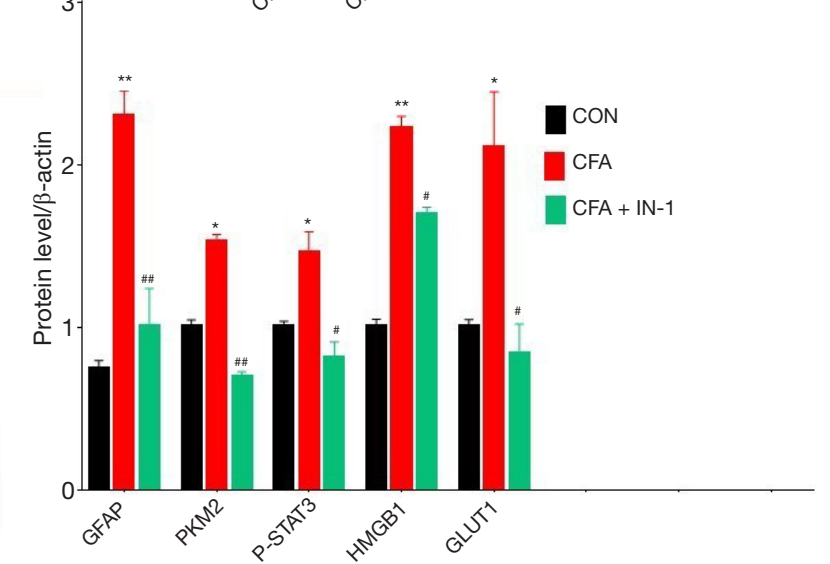

Figure 4 Intrathecal injection of PKM2-IN-1 reduced CFA induced mechanical hyperalgesia and the expression of related proteins. (A) Intrathecal PKM2-IN-1 reversed mechanical hyperalgesia induced by CFA, ${ }^{* *}, \mathrm{P}<0.01$ compared with $C O N,{ }^{*}, \mathrm{P}<0.05,{ }^{\# \#}, \mathrm{P}<0.01$ compared with CFA, $n=6$ rats/group. (B) PRP and PKM2-IN-1 significantly decreased lactate production. $\mathrm{N}=4$. (C) Western blot showed PKM2-IN-1 partially reversed the CFA induced increase in GFAP, PKM2, p-STAT3, GLUT1 and HMGB1 protein levels in the spinal cord. (D) The bar graphs showed the ratios of GFAP, PKM2, p-STAT3, GLUT1, and HMGB1 to $\beta$-actin. Data are means $\pm \mathrm{SEM} \mathrm{N}=4$. *, $\mathrm{P}<0.05$ compared with $\mathrm{CON}$; ${ }^{* *} \mathrm{P}<0.01$, compared with $\mathrm{CON} ;{ }^{\#}, \mathrm{P}<0.05,{ }^{\#}, \mathrm{P}<0.01$ vs. CFA. PRP, platelet-rich plasma; CFA, complete Freund's adjuvant; IN1, PKM2-IN-1.

PRP, the expression of PKM2 protein was significantly decreased compared with the LPS group. (Figure 5E) (scale bar $=100 \mu \mathrm{m}$, green: GFAP, red: PKM2). We also detected the expression of GFAP, PKM2, p-STAT3, GLUT1, and HMGB1 by Western blotting, which showed LPS significantly activated PKM2-mediated glycolysis, while PRP treatment significantly decreased it compared with the LPS group (Figure $5 F, G$ ). These results show that astrocytes' glycolytic level increases after LPS stimulation; PRP, however, can inhibit these effects.

\section{Inbibition of PKM2 by PKM2-IN-1 attenuates LPS-activated glycolysis (Figure 6)}

Pyruvate kinase plays a crucial role in glycolysis; with an in-depth study, it has been discovered that increased glucose uptake and glycolytic flux. In turn, the promotion of PKM2 translocation into the nucleus and exerting different effects in combination with various transcription factors. It is known there are STAT3 binding elements in the gene promoter region of the astrocyte marker GFAP. Earlier results have shown that CFA leads to a significant up-regulation of phosphorylated STAT3; therefore, we will investigate whether PKM2 can activate astrocytes by activating STAT3 subsequently. We used an inflammation model in which astrocytes were stimulated with LPS $(100 \mathrm{ng} / \mathrm{mL})$ in vitro. The results of immunofluorescence staining indicated the expression of PKM2 and p-STAT3 was significantly enhanced in the nucleus after LPS treatment, while $3 \mu \mathrm{mol} / \mathrm{L}$ PKM2-IN-1, an inhibitor of PKM2, significantly decreased the effect (Figure 6A). Also, we verified by Western blotting and got consistent results (Figure $6 B, C$ ). We further examined the localization of PKM2 in primary astrocytes after LPS stimulation in vitro. Immunofluorescence analysis was performed on astrocytes after LPS stimulation. Similarly, PKM2 was located 
A

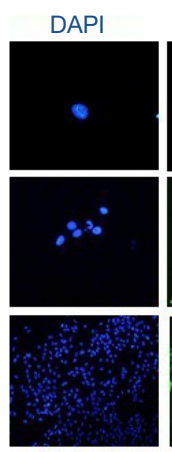

$\mathrm{E}$

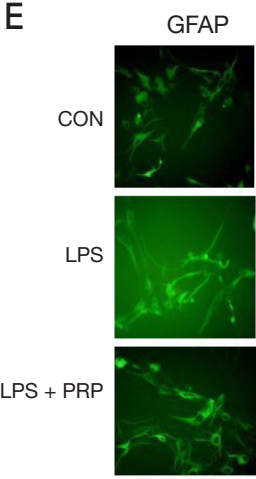

GFAP
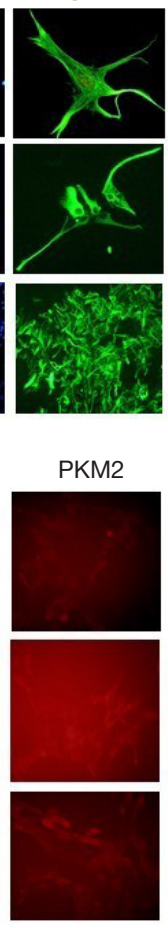
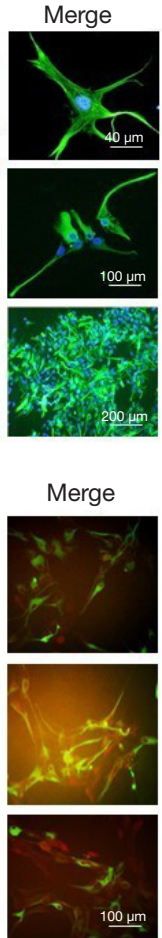

B

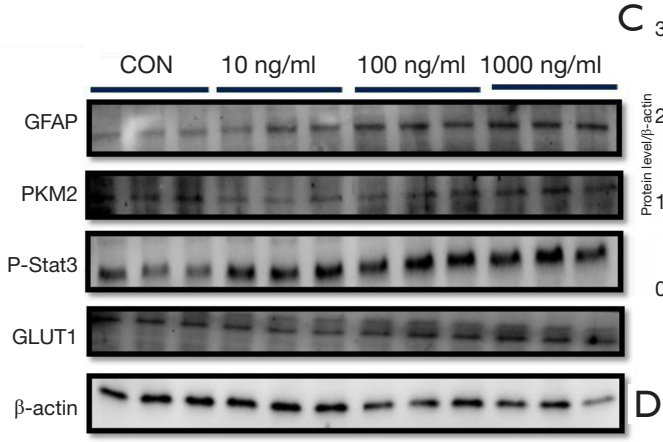

$\mathrm{F}$

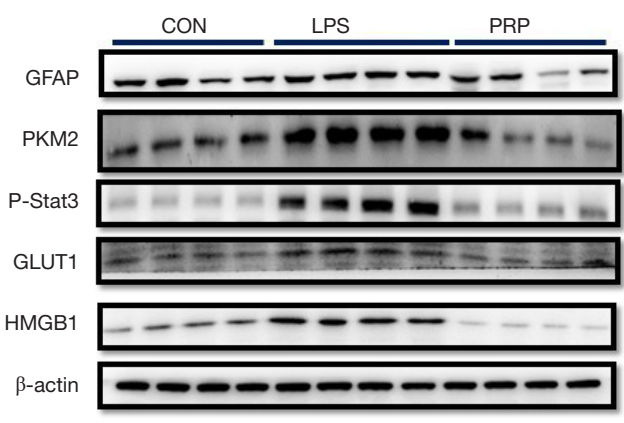

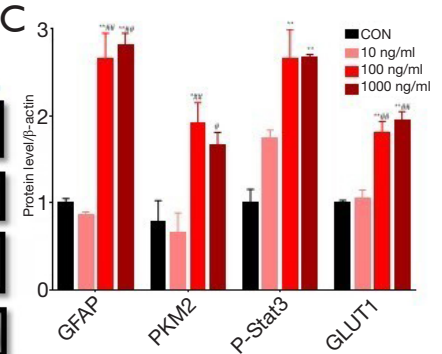

G
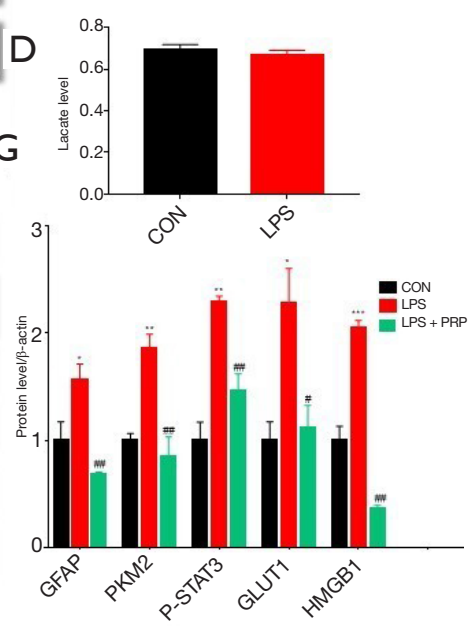

Figure 5 PRP inhibits LPS-induced glycolytic levels in primary astrocytes (A) Astrocytes purified by repeated passages were found using GFAP and DAPI immunofluorescence double labeling. Scale bars: 40 (top) 100 (middle) and $200 \mu \mathrm{m}$ (bottom). (B) primary astrocytes were stimulated with LPS $(10,100,1,000 \mathrm{ng} / \mathrm{mL})$ for 12 hours. Western blot showed that GFAP, PKM2, p-STAT3, and GLUT1 were significantly increased after LPS stimulation. (C) The bar graphs showed the ratios of GFAP, PKM2, p-STAT3, and GLUT1 to $\beta$-actin at each concentration. Data are means $\pm \operatorname{SEM}\left(\mathrm{n}=4,{ }^{*}, \mathrm{P}<0.05,{ }^{* *}, \mathrm{P}<0.01\right.$, significantly different from $\mathrm{CON},{ }^{*}, \mathrm{P}<0.05,{ }^{\#}, \mathrm{P}<0.01$, significantly different from $10 \mathrm{ng} / \mathrm{mL}$ ). (D) the release of lactic acid did not increase; (E) Immunofluorescence shows that PRP inhibits the expression of GFAP (green) and PKM2 (red). Scale bar: $100 \mu \mathrm{m}$. n=3. (F) Western blot showed GFAP, PKM2 was significantly decreased after PRP treatment as well as p-STAT3, GLUT1and HMGB1. (G) The bar graphs showed the ratios of GFAP, PKM2, p-STAT3, GLUT1, and HMGB1 to $\beta$-actin. Data are means $\pm \operatorname{SEM}\left(\mathrm{n}=4,{ }^{*}, \mathrm{P}<0.05,{ }^{* *}, \mathrm{P}<0.01,{ }^{* * *}, \mathrm{P}<0.001\right.$ significantly different from $\mathrm{CON},{ }^{*}, \mathrm{P}<0.05,{ }^{* \#}, \mathrm{P}<0.01$, significantly different from LPS). PRP, platelet-rich plasma; LPS, lipopolysaccharide.

within the nucleus 12 hours after LPS administration. However, there was no change in the CON and PKM2IN-1 groups. (Figure 6D). Besides, Western blot analysis was performed on astrocytes after LPS stimulation, as predicted, the expression of PKM2 was up-regulated in the nucleus (Figure 6E,F) while decreased in the cytoplasm at 12 hours after LPS exposure (Figure 6G,H). These data suggest LPS stimulation of astrocytes up-regulates PKM2mediated glycolysis, while PKM2-IN-1 can inhibit PKM2 to attenuate LPS-activated glycolysis.

\section{Discussion}

Inflammatory pain is a chronic pain mediated by inflammatory or pain-causing factors, with pathological manifestations, including redness and swelling of inflammation and pathological pain characteristics including hyperalgesia, paresthesia, and spontaneous pain (32). In this study, the animal model of inflammatory pain was reproduced by injecting CFA into the rat's foot. After four hours of CFA administration, the paw withdrawal reflex threshold of mechanical stimulation in rats was significantly reduced, indicating that this animal model of inflammatory pain was successfully reproduced. However, after PRP treatment, the paw withdrawal reflex threshold of mechanical stimulation in rats with inflammatory pain was significantly increased, which suggests that PRP has a specific alleviating effect on inflammatory pain induced 
A

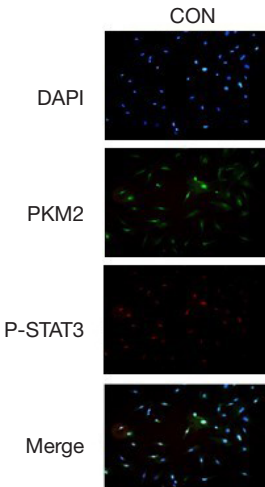

D

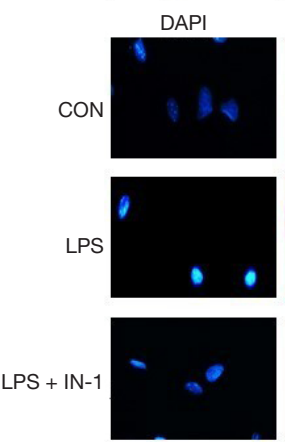

LPS

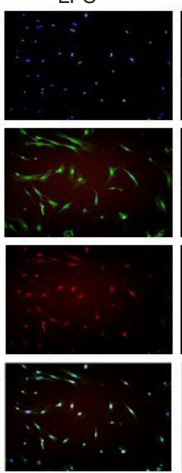

PKM2
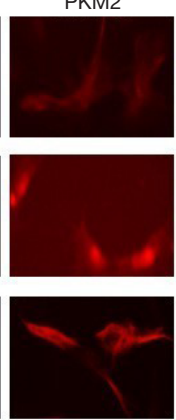

LPS + IN-1

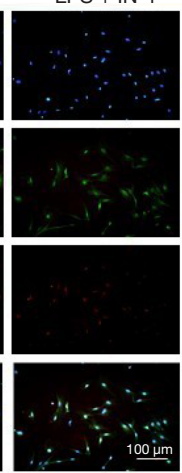

Merge
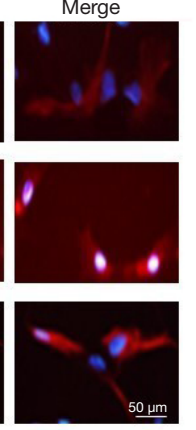

B
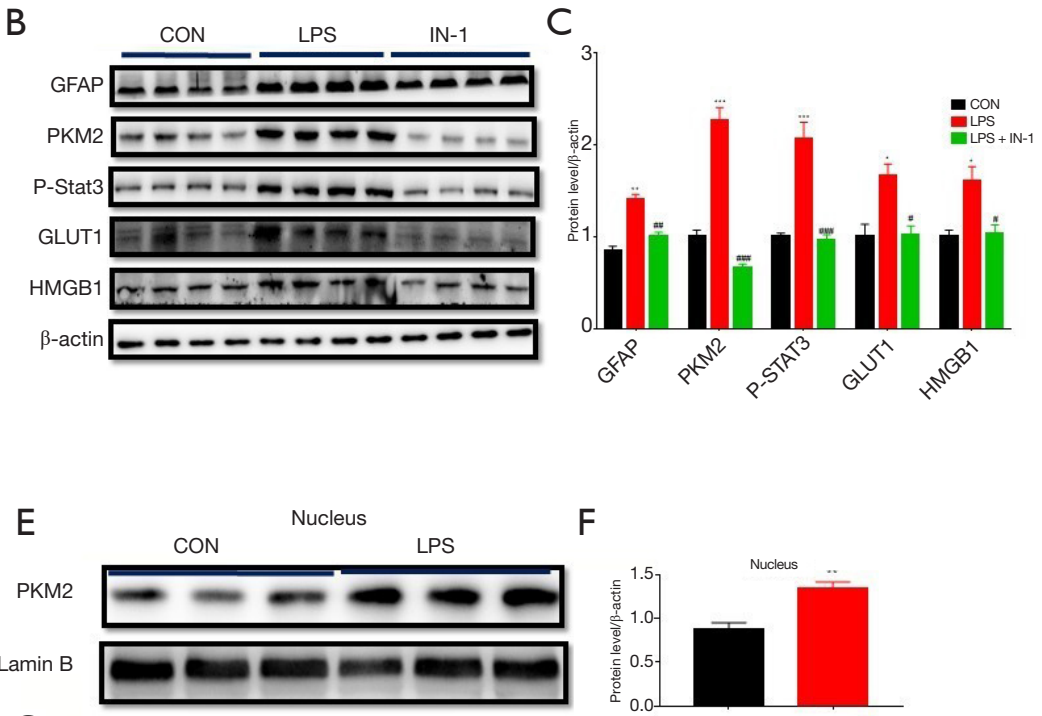

G PKM2

$\beta$-actin

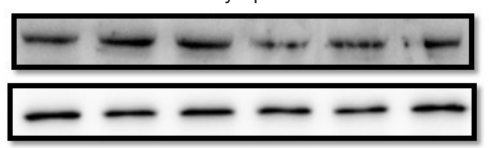

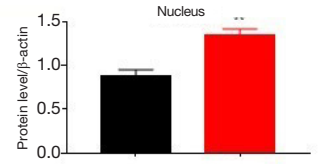

$\mathrm{H}$

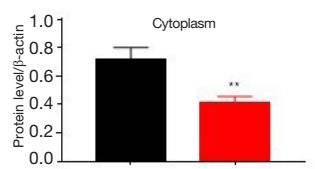

Figure 6 Inhibition of PKM2 by PKM2-IN-1 attenuates the role of PKM2 in LPS-activated glycolysis. (A) Immunofluorescence showed that PKM2-IN-1, an inhibitor of PKM2, attenuated the expression of PKM2 (green) and p-STAT3 (red) in the nucleus. Scale bar: $100 \mu \mathrm{m}$. n=3. (B) Western blot showed GFAP, PKM2 was significantly decreased after PKM2-IN-1 treatment as well as p-STAT3, GLUT1 and HMGB1. (C) The bar graphs showed the ratios of GFAP, PKM2, p-STAT3, GLUT1, and HMGB1 to $\beta$-actin. Data are means \pm SEM ( $n=4$, ${ }^{*}, \mathrm{P}<0.05,{ }^{* *}, \mathrm{P}<0.01,{ }^{* * *}, \mathrm{P}<0.001$ significantly different from CON, ${ }^{*}, \mathrm{P}<0.05,{ }^{* \#}, \mathrm{P}<0.01$, , ${ }^{\# \#}, \mathrm{P}<0.001$ significantly different from LPS). (D) Immunofluorescence showed that PKM2 was largely redistributed to the nucleus, while cytoplasm numbers were reduced in response to LPS. However, PKM2 was mainly distributed in the cytoplasm in the CON and IN-1 group. Scale bar: $50 \mu \mathrm{m} . \mathrm{n}=3$ (E-H) Nuclear and cytoplasmic proteins of PKM2 were detected by western blotting. Quantitative analysis of the intensity of PKM2 to Lamin B (E,F)/ $\beta$-actin $(\mathrm{G}, \mathrm{H})$. Data are means \pm SEM ( $\mathrm{n}=3$; **, $\mathrm{P}<0.01$, significantly different from CON). LPS, lipopolysaccharide; IN-1, PKM2-IN-1.

by CFA. However, the mechanism of action of PRP in relieving inflammatory pain is still unclear.

PRP is an autologous blood product, so there is no immune rejection and no risk of disease transmission when treated with PRP; moreover, PRP has the advantages of the simple preparation process, small patient wound surface, low treatment cost, and biological therapeutic potential, and has been widely used in various clinical fields (33-35). Studies have shown that PRP injection can promote functional improvement and relieve pain by inhibiting the inflammatory response. The rabbit tenocyte in vitro model and rabbit Achilles tendon in vivo study by Zhang et al. demonstrated that the anti-inflammatory effect of PRP is closely related to HGF (36). Meanwhile, PRP can up-regulate the expression of hepatocyte growth factor and
TNF- $\alpha$ of which hepatocyte growth factor and TNF- $\alpha$ can inhibit the inflammatory response by blocking the expression of NF-kB (37). Studies conducted on both glial cells and pain have demonstrated that pharmacological inhibition of the inflammatory response in the central nervous system not only down-regulates the high expression of inflammatory factors but also inhibits the activation and proliferation of glial cells. Also, it has been found to alleviate neuropathic pain significantly.

Further, In the inflammatory pain model of rats injected with CFA in the hind paw, the improvement of pain behavior was accompanied by the inhibition of activated glial cells in the spinal dorsal horn. From these findings, we hypothesize that PRP can significantly inhibit or reduce the hyperreactivity and pain response of spinal dorsal 
horn neurons induced by inflammatory responses during spinal glial cells' activation phase. Our results suggest that intrathecal PRP can alleviate CFA induced pain in rats, inhibit the activation of astrocytes, and downregulate the high expression of PKM2, p-STAT3 and HMGB1. Studies have shown that fibroblast growth factor $(38,39)$ and hepatocyte growth factor $(40,41)$ can reduce pain by inhibiting the activation of astrocytes in the spinal cord; and PRP contains various growth factors, including fibroblast growth factor and hepatocyte growth factor (42), so a longterm higher concentration of PRP acts on cells or may make such inhibitory effects more obvious

Traditional studies have suggested that peripheral and central sensitization causes chronic pain due to changes in neuronal plasticity. However, recent studies have found that glial cells, especially astrocytes, play an essential role in maintaining chronic pain $(43,44)$. The nociceptive stimulus signals were transmitted to the spinal cord, and high central sites through primary afferent fibers after tissue injury can activate astrocytes. Then, the activated glial cells were transformed from oxidative phosphorylation to aerobic glycolysis to meet the increased energy demand metabolic mode (45). The activation of these cells resulted in the release of various inflammatory cytokines that induce pain and promote neuronal sensitization (46), thus further aggravating central sensitization.

However, pain can be reduced by inhibiting glial cells $(47,48)$. Previous studies have shown that metabolic activity and glucose utilization in the spinal dorsal horn is significantly improved in CCI pain model rats (49). A recently published study examined the potential role of pyruvate kinase isoenzyme M2 (PKM2) in neuropathic pain and showed that inhibition of PKM2 expression effectively reduced neuropathic pain and inflammatory responses in a rat model by modulating ERK and STAT3 signaling pathways (50). With the deepening of research, PKM2 was found to play an essential role between metabolism and inflammation (51). Therefore, whether PKM2 takes part in inflammatory pain has attracted our greatest attention. Our study found that PKM2 expression was increased in the spinal cord after CFA injection and was accompanied by altered pain behavior. Simultaneously, we detected an increase in GLUT1 and glycolysis product lactic acid levels, which may be due to increased glucose uptake and increased glycolysis activity (52). These results show that PKM2 may be involved in the development of CFA inflammatory pain. To demonstrate our speculation, we performed intrathecal injection of PKM2-IN-1, an inhibitor of PKM2, in rats, and the results showed an inhibitor of PKM2 markedly alleviated pain hypersensitivity and glycolysis level caused by CFA. However, we found inconsistent results in LPS-treated primary astrocytes. Astrocytes are highly glycolytic cells that release substantial amounts of lactate in the extracellular space. In the brain, surrounding neurons can absorb these lactates, converted back to pyruvate, and used as an energy substrate to maintain high energy requirements (53). Although LPS significantly increased astrocyte glucose utilization in this study (54), no increase in lactate release was observed. These findings were consistent with data from other combinations of inflammatory cytokines treated for 48 hours (55). The findings are possible because they increased glucose utilization, increased TCA cycle activity, increased PPP activity (both combined), and decreased glycogen content, which can be stored as the energy released from lactate without significantly affecting lactate release (56).

Activation of the astrocyte marker GFAP may be associated with JAK/Stat-mediated enhanced expression of the GFAP gene. A potential STAT3 binding site (TTCCGAGAA) was found at the rat GFAP promoter (57). The JAK2/STAT3 pathway is a cascade of astrocyte (AST) responses to tissue or nerve injury (58). In general, cytokines activate JAK2 kinase by binding to cytokine receptors and then activate STAT3, and activated STAT3 enters the nucleus to regulate the expression of the GFAP gene. These activated and translocated into the nucleus STAT3, combined with specific sequences, can regulate the expression of GFAP target genes and exert its transcriptional regulation. Some studies have shown that blocking the JAK-STAT3 pathway can effectively relieve pain (59). Our study found that activated STAT3 expression increased after CFA and PRP could inhibit its expression. Recent studies have also reported HMGB1 also plays a vital role in chronic pain models in rodents, including the spinal dorsal horn and dorsal root ganglion (DRG) in animal models of cancer pain and neuropathic pain (60). Under conditions of inflammation and stress, HMGB1 can also be actively secreted from nerve cells and glial cells, which, in turn, is involved in the development and maintenance of pain (61). Under pain conditions, astrocytes in the spinal dorsal horn are widely activated, which in turn causes the production and release of various inflammatory mediators, and the secretion of HMGB1 can also trigger the release of cytokines from inflammatory cells, causing positive feedback, which further causes the release of HMGB1 


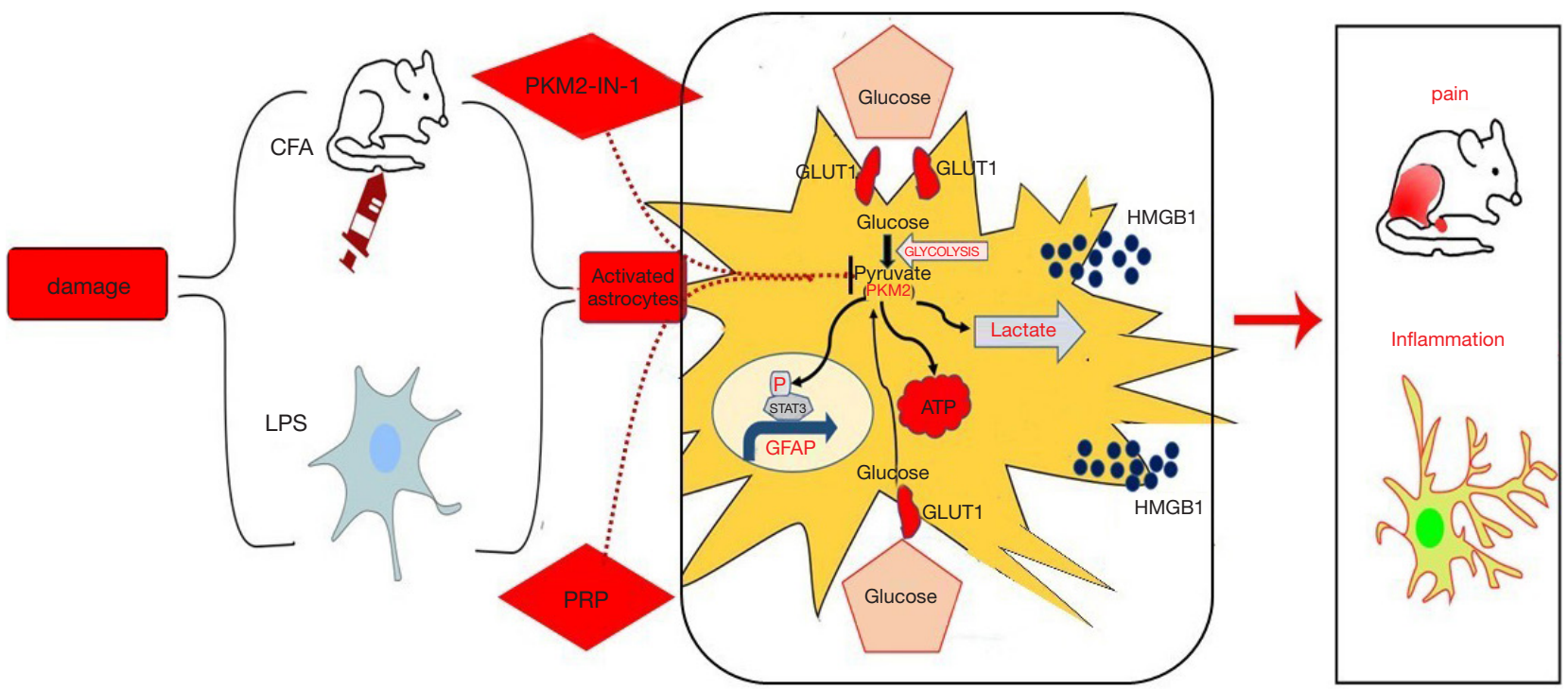

Figure 7 The primary mechanism of PRP improves chronic inflammatory pain. Astrocyte activation after CFA or LPS stimulation. The uptake of more glucose by activated astrocytes increases aerobic glycolysis and promotes sustained activation of living astrocytes and secretion of HMGB1 through the STAT3 signaling pathway. PRP ameliorates inflammatory pain by inhibiting PKM2-mediated aerobic glycolysis and the STAT3 signaling pathway to reduce activation of astrocytes and HMGB1 secretion. PRP, platelet-rich plasma; CFA, complete Freund's adjuvant. LPS, lipopolysaccharide.

and the maintenance of the inflammatory cascade (62). In our study, the expression of HMGB1 protein in the spinal cord of CFA rats was significantly up-regulated, while inhibition of astrocytes could reduce the expression of HMGB1 and produce an analgesic effect, suggesting that the mechanical hyperalgesia of inflammatory pain was significantly inhibited after reducing the level of HMGB1 in the spinal cord. Thus, our results suggest that spinal HMGB1 takes part in the maintenance of mechanical hyperalgesia in inflammatory pain.

\section{Conclusions}

In conclusion, our data showed CFA induced astrocyte activation in the spinal dorsal horn, PKM2-mediated glycolysis was significantly increased, and PRP effectively inhibited the inflammatory response and HMGB1 expression by regulating PKM2-mediated glycolysis and STAT3 signaling-mediated astrocyte activation, thereby alleviating CFA induced pain in rats (Figure 7).

\section{Acknowledgments}

Funding: This work was supported by the National Natural
Science Foundation of China (81701098 to XWM), Jiangsu Provincial Medical Youth Talents Program (QNRC2016740 to LNW), Jiangsu Provincial Medical Innovation Team (CXTDA2017043 to FHJ), and Suzhou people's Livelihood Science and technology project (SYS201738 to XWM).

\section{Footnote}

Reporting Checklist: The authors have completed the ARRIVE reporting checklist. Available at http://dx.doi. org/10.21037/atm-20-6502

Data Sharing Statement: Available at http://dx.doi. org/10.21037/atm-20-6502

Conflicts of Interest: All authors have completed the ICMJE uniform disclosure form (available at http://dx.doi. org/10.21037/atm-20-6502). The authors have no conflicts of interest to declare.

Ethical Statement: The authors are accountable for all aspects of the work in ensuring that questions related to the accuracy or integrity of any part of the work are appropriately investigated and resolved. Experimental 
protocols were approved by the Institutional Animal Care and Use Committee of Soochow University (NO.: 2017185). All experiments were performed following the National Institutes of Health Guide for the Care and Use of Laboratory Animals and the guidelines of the International Association for the Study of Pain.

Open Access Statement: This is an Open Access article distributed in accordance with the Creative Commons Attribution-NonCommercial-NoDerivs 4.0 International License (CC BY-NC-ND 4.0), which permits the noncommercial replication and distribution of the article with the strict proviso that no changes or edits are made and the original work is properly cited (including links to both the formal publication through the relevant DOI and the license). See: https://creativecommons.org/licenses/by-nc-nd/4.0/.

\section{References}

1. Chen O, Donnelly CR, Ji RR. Regulation of pain by neuro-immune interactions between macrophages and nociceptor sensory neurons. Curr Opin Neurobiol 2020;62:17-25.

2. Qin Z, Wu J, Xu C, et al. Long-term effects of acupuncture for chronic prostatitis/chronic pelvic pain syndrome: systematic review and single-arm meta-analyses. Ann Transl Med 2019;7:113.

3. Zhang ZJ, Jiang BC, Gao YJ. Chemokines in neuron-glial cell interaction and pathogenesis of neuropathic pain. Cell Mol Life Sci 2017;74:3275-91.

4. Hossain MZ, Unno S, Ando H, et al. Neuron-Glia Crosstalk and Neuropathic Pain: Involvement in the Modulation of Motor Activity in the Orofacial Region. Int J Mol Sci 2017;18:2051.

5. Cohen SP, Mao J. Neuropathic pain: mechanisms and their clinical implications. BMJ 2014;348:f7656.

6. Penas C, Navarro X. Epigenetic Modifications Associated to Neuroinflammation and Neuropathic Pain After Neural Trauma. Front Cell Neurosci 2018;12:158.

7. Matsuda M, Huh Y, Ji RR. Roles of inflammation, neurogenic inflammation, and neuroinflammation in pain. J Anesth 2019;33:131-9.

8. O'Neill LA, Hardie DG. Metabolism of inflammation limited by AMPK and pseudo-starvation. Nature 2013;493:346-55.

9. Nakagawa T, Lanaspa MA, Millan IS, et al. Fructose contributes to the Warburg effect for cancer growth. Cancer Metab 2020;8:16.
10. Krawczyk CM, Holowka T, Sun J, et al. Toll-like receptorinduced changes in glycolytic metabolism regulate dendritic cell activation. Blood 2010;115:4742-9.

11. Orihuela R, McPherson CA, Harry GJ. Microglial M1/ M2 polarization and metabolic states. Br J Pharmacol 2016;173:649-65.

12. Yang L, Xie M, Yang M, et al. PKM2 regulates the Warburg effect and promotes HMGB1 release in sepsis. Nat Commun 2014;5:4436.

13. Brandi J, Cecconi D, Cordani M, et al. The antioxidant uncoupling protein 2 stimulates hnRNPA2/B1, GLUT1 and PKM2 expression and sensitizes pancreas cancer cells to glycolysis inhibition. Free Radic Biol Med 2016;101:305-16.

14. Dayton TL, Jacks T, Vander Heiden MG. PKM2, cancer metabolism, and the road ahead. EMBO Rep 2016;17:1721-30.

15. Zhang Y, Chen K, Sloan SA, et al. An RNA-sequencing transcriptome and splicing database of glia, neurons, and vascular cells of the cerebral cortex. J Neurosci 2014;34:11929-47.

16. Zhang Q, Bian G, Chen P, et al. Aldose Reductase Regulates Microglia/Macrophages Polarization Through the cAMP Response Element-Binding Protein After Spinal Cord Injury in Mice. Mol Neurobiol 2016;53:662-76.

17. Walkowiak J, Banasiewicz T, Krokowicz P, et al. Fecal pyruvate kinase (M2-PK): a new predictor for inflammation and severity of pouchitis. Scand J Gastroenterol 2005;40:1493-4.

18. Lv L, Xu YP, Zhao D, et al. Mitogenic and oncogenic stimulation of K433 acetylation promotes PKM2 protein kinase activity and nuclear localization. Mol Cell 2013;52:340-52.

19. Yang W, Xia Y, Ji H, et al. Nuclear PKM2 regulates betacatenin transactivation upon EGFR activation. Nature 2011;480:118-22.

20. Gao X, Wang H, Yang JJ, et al. Pyruvate kinase M2 regulates gene transcription by acting as a protein kinase. Mol Cell 2012;45:598-609.

21. Dominguez E, Rivat C, Pommier B, et al. JAK/STAT3 pathway is activated in spinal cord microglia after peripheral nerve injury and contributes to neuropathic pain development in rat. J Neurochem 2008;107:50-60.

22. Taga T, Fukuda S. Role of IL-6 in the neural stem cell differentiation. Clin Rev Allergy Immunol 2005;28:249-56.

23. Lee HS, Han J, Lee SH, et al. Meteorin promotes the formation of GFAP-positive glia via activation of the JakSTAT3 pathway. J Cell Sci 2010;123:1959-68. 
24. Branford OA, Klass BR, Grobbelaar AO, et al. The growth factors involved in flexor tendon repair and adhesion formation. J Hand Surg Eur Vol 2014;39:60-70.

25. Kang YH, Jeon SH, Park JY, et al. Platelet-rich fibrin is a Bioscaffold and reservoir of growth factors for tissue regeneration. Tissue Eng Part A 2011;17:349-59.

26. Lee JK, Lee S, Han SA, et al. The effect of plateletrich plasma on the differentiation of synovium-derived mesenchymal stem cells. J Orthop Res 2014;32:1317-25.

27. Prado J, Popovceleketic J, Steenlouws C, et al. Development of Recombinant Proteins to Treat Chronic Pain. J Vis Exp 2018;2018.

28. Xing F, Zhang W, Wen J, et al. TLR4/NF-кB signaling activation in plantar tissue and dorsal root ganglion involves in the development of postoperative pain. Mol Pain 2018;14:1744806918807050.

29. Freymiller EG, Aghaloo TL. Platelet-rich plasma: ready or not? J Oral Maxillofac Surg 2004;62:484-8.

30. Xing F, Kong C, Bai L, et al. CXCL12/CXCR4 signaling mediated ERK1/2 activation in spinal cord contributes to the pathogenesis of postsurgical pain in rats. Mol Pain 2017;13:1744806917718753.

31. Monfett M, Harrison J, Boachie-Adjei K, et al. Intradiscal platelet-rich plasma (PRP) injections for discogenic low back pain: an update. Int Orthop 2016;40:1321-8.

32. Zhang T, Zhang N, Zhang R, et al. Preemptive intrathecal administration of endomorphins relieves inflammatory pain in male mice via inhibition of p38 MAPK signaling and regulation of inflammatory cytokines. J Neuroinflammation 2018;15:320.

33. Guo SC, Tao SC, Yin WJ, et al. Exosomes derived from platelet-rich plasma promote the re-epithelization of chronic cutaneous wounds via activation of YAP in a diabetic rat model. Theranostics 2017;7:81-96.

34. Liao AX, A JXL, A SHL, et al. Allogeneic Platelet-Rich Plasma Therapy as an Effective and Safe Adjuvant Method for Chronic Wounds. J Surg Res 2020;246:284-91.

35. Cakin MC, Ozdemir B, Kaya-Dagistanli F, et al. Evaluation of the in vivo wound healing potential of the lipid fraction from activated platelet-rich plasma. Platelets 2020;31:513-20.

36. Zhang J, Middleton KK, Fu FH, et al. HGF mediates the anti-inflammatory effects of PRP on injured tendons. PLoS One 2013;8:e67303.

37. Molecular basis of anti-inflammatory action of plateletrich plasma on human chondrocytes: Mechanisms of NF$\kappa \mathrm{B}$ inhibition via HGF. J Cell Physiol 2010;225:757-66.

38. Lin YL, Kuo HS, Lo MJ, et al. Treatment with nerve grafts and aFGF attenuates allodynia caused by cervical root transection injuries. Restor Neurol Neurosci 2011;29:265-74.

39. Forouzanfar F, Amin B, Ghorbani A, et al. New approach for the treatment of neuropathic pain: Fibroblast growth factor 1 gene-transfected adipose-derived mesenchymal stem cells. Eur J Pain 2018;22:295-310.

40. $\mathrm{Hu} \mathrm{C}, \mathrm{Lu} \mathrm{Y}$, Chen X, et al. Gene transfer of a naked plasmid (pUDK-HGF) encoding human hepatocyte growth factor attenuates skin/muscle incision and retraction-induced chronic post-surgical pain in rats. Eur J Pain 2018;22:961-72.

41. Nho B, Lee J, Lee J, et al. Effective control of neuropathic pain by transient expression of hepatocyte growth factor in a mouse chronic constriction injury model. FASEB J 2018;32:5119-31.

42. Baria M, Vasileff WK, Miller M, et al. Cellular Components and Growth Factor Content of Platelet-Rich Plasma With a Customizable Commercial System. Am J Sports Med 2019;47:1216-22.

43. Luongo L, Maione S, Di Marzo V. Endocannabinoids and neuropathic pain: focus on neuron-glia and endocannabinoid-neurotrophin interactions. Eur J Neurosci 2014;39:401-8.

44. Ji RR, Donnelly CR, Nedergaard M. Astrocytes in chronic pain and itch. Nat Rev Neurosci 2019;20:667-85.

45. Rahman MH, Jha MK, Suk K. Evolving Insights into the Pathophysiology of Diabetic Neuropathy: Implications of Malfunctioning Glia and Discovery of Novel Therapeutic Targets. Curr Pharm Des 2016;22:738-57.

46. Chichorro JG, Lorenzetti BB, Zampronio AR. Involvement of bradykinin, cytokines, sympathetic amines and prostaglandins in formalin-induced orofacial nociception in rats. Br J Pharmacol 2004;141:1175-84.

47. Xu F, Li Y, Li S, et al. Complete Freund's adjuvantinduced acute inflammatory pain could be attenuated by triptolide via inhibiting spinal glia activation in rats. J Surg Res 2014;188:174-82.

48. Zucoloto AZ, Manchope MF, Borghi SM, et al. Probucol Ameliorates Complete Freund's Adjuvant-Induced Hyperalgesia by Targeting Peripheral and Spinal Cord Inflammation. Inflammation 2019;42:1474-90.

49. Mao J, Price DD, Coghill RC, et al. Spatial patterns of spinal cord [14C]-2-deoxyglucose metabolic activity in a rat model of painful peripheral mononeuropathy. Pain 1992;50:89-100.

50. Wang B, Liu S, Fan B, et al. PKM2 is involved in neuropathic pain by regulating ERK and STAT3 activation 
in rat spinal cord. J Headache Pain 2018;19:7.

51. Alves-Filho JC, Palsson-McDermott EM. Pyruvate Kinase M2: A Potential Target for Regulating Inflammation. Front Immunol 2016;7:145.

52. Prado-Garcia H, Campa-Higareda A, Romero-Garcia S. Lactic Acidosis in the Presence of Glucose Diminishes Warburg Effect in Lung Adenocarcinoma Cells. Front Oncol 2020;10:807.

53. Magistretti PJ. Neuron-glia metabolic coupling and plasticity. J Exp Biol 2006;209:2304-11.

54. Cidad P, Garcia-Nogales P, Almeida A, et al. Expression of glucose transporter GLUT3 by endotoxin in cultured rat astrocytes: the role of nitric oxide. J Neurochem 2001;79:17-24.

55. Belanger M, Allaman I, Magistretti PJ. Differential effects of pro- and anti-inflammatory cytokines alone or in combinations on the metabolic profile of astrocytes. J Neurochem 2011;116:564-76.

56. Gavillet M, Allaman I, Magistretti PJ. Modulation of astrocytic metabolic phenotype by proinflammatory cytokines. Glia 2008;56:975-89.

Cite this article as: Wei X, Jin XH, Meng XW, Hua J, Ji FH, Wang LN, Yang JP. Platelet-rich plasma improves chronic inflammatory pain by inhibiting PKM2-mediated aerobic glycolysis in astrocytes. Ann Transl Med 2020;8(21):1456. doi: 10.21037/atm-20-6502
57. Sharma KD, Schaal D, Kore RA, et al. Glioma-derived exosomes drive the differentiation of neural stem cells to astrocytes. PLoS One 2020;15:e0234614.

58. Yamauchi K, Osuka K, Takayasu M, et al. Activation of JAK/STAT signalling in neurons following spinal cord injury in mice. J Neurochem 2006;96:1060-70.

59. Maeda T, Kiguchi N, Kobayashi Y, et al. Leptin derived from adipocytes in injured peripheral nerves facilitates development of neuropathic pain via macrophage stimulation. Proc Natl Acad Sci U S A 2009;106:13076-81.

60. Tong W, Wang W, Huang J, et al. Spinal high-mobility group box 1 contributes to mechanical allodynia in a rat model of bone cancer pain. Biochem Biophys Res Commun 2010;395:572-6.

61. Liu C, Yang J, Liu H, et al. miR-300 mitigates cancerinduced bone pain through targeting HMGB1 in rat models. Genes Genomics 2020;42:309-16.

62. Andersson U, Erlandsson-Harris H, Yang H, et al. HMGB1 as a DNA-binding cytokine. J Leukoc Biol 2002;72:1084-91. 